\title{
A Unit Plane Edge on-off Slope Algorithm Based Fast LTVR Res- toration Analysis
}

\author{
K. Praveen Kumar ${ }^{1}$, C. Venkata Narasimhulu' ${ }^{2}$, K. Satya Prasad ${ }^{3}$ \\ ${ }^{I}$ Research Scholar, Department of ECE, JNTUK, Kakinada \\ ${ }^{2}$ Department of ECE, GCET, Hyderabad \\ ${ }^{3}$ Department of ECE, KL University, Vijayawada \\ * Correspondence Email:praveen416.kumar@gmail.com
}

\begin{abstract}
This research paper presents a Fast LTVR (Localized Total Variation Regularized) method for restoring the degraded images by white noise, while preserving the image edge details in a constructed unit plane edge model through a Unit Plane Edge ON-OFF Slope algorithm. The noisy image contains two details; one with high noise and the other with edge fined details. The edge fine details are restored using ON-OFF Slope algorithm. The denoised image and the edge fine details are used to reconstruct the final restored image. A Unit Plane Edge restoration method is proposed in this research work to estimate the edge-mapping with the fine details. Simulation results of proposed work shows an effective image restoration algorithm comparatively with different filter based restoration methods.
\end{abstract}

Keywords: : Image denoising; filter; ON-OFF; edge; restoration

\section{Introduction}

Image restoration has been in researching quite thoroughly to develop an effective denoising method that can retain the original image by removing the complete noise. During the process of image restoration, the edge is so precious, which is a challenging task in image processing techniques like compression, denoising and restoration. The image denoising technique so far implemented [1-5] could able to reconstruct the image area which is beyond the edges, and could able to restore with less quality and is the first challenging task in the present scenario of this research work. And the restoration of edge pixels using TV formulation is the second challenging task, which accomplishes the primal-dual method and min-max optimization, are the approaches implemented in this research work.

\section{Previous Work}

Form the literature survey, the methods to restore an image include non-linear and linear methods, for the fined and un-fined regions of the noised image, which was proposed in [4], [5] and [6] using different methods. The authors G. Gilboa, N. Sochen, and Y. Zeevi in [4], utilises a Adaptive Total Variation (ATV) method to denoise the fine regions of image and could able adaptively estimate the image reconstruction. The method in [5], utilises a NonLocal Means method (NLM) to eliminate the noise smoothen areas. The method in [6], utilises a inverse filtering method to eliminate the additive noise present in the smooth and non-smooth areas, which is a best technique for the fine image denoising technique in spatial [7-9] and frequency [10-11] domains. Moving further, [12-13] wavelet denoising techniques have been implementing in the present scenario to reduce the computation time [14] and precision of denoising [15], one among them is Lifting-based wavelet domain adaptive Wiener filter (LBWDWF) [1] method to improve the image restoration by providing an increase in the computation time compared to traditional wavelets [16] [17]. Second among them is [8], to eliminate the neighbourhood local variance around the pixel region. Further subjective enhancement techniques proposed in have improved in estimating the noise variance in spatial and frequency domain.

The similar approach related to Total Variation (TV) proposed a minimal energy function to reduce the noise variation and total variation of the image adaptively. An approach in (FDWF)[2] Frequency Domain Weiner Filter provides a spectra power estimation through filter-based denoising method. In Edge map and Wiener Filter (EWF) [3], the denoising of details are preserved by reducing the noise levels.

\section{Proposed Work}

In this research work, comparing with the (LBWDWF)[1], (FDWF)[2], (EWF) [3], (ATV)[4] and (NLM)[5], the following are the key features:
a. increased image pixel preservation
b. fasten the computation performance
c. increased the noise estimation
d. a strong denoising in the smooth regions, a variant TV method is proposed
e. to preserve the fine and edge details during the higher noise areas

The proposed Unit Plane Edge ON-OFF Slope algorithm is used to de-noise the edge details by preserving the neighbour pixels in the regions of image restorations. The image denoised area is considered as fine and non-fine regions. Across the fine regions, the unit threshold value restoration is estimated and across the nonfine regions, the plane threshold value restoration is estimated. And across the edges, ON-OFF slope threshold values are applied 
to estimate the edge details in fine and non-fine regions. A new TV based method is derived and implemented in proposed method to visualise the fine and non-fine regions of the image. TV based method utilizes a corner-edge map decision to perform edge fining during the restoration process by taking the strong and weak threshold values across the fine and edge regions.

Total Variational (TV) models are widely used in this area of work and in analyzing image restoration. Fundamental image restoration problem define denoising, which takes a step ahead in computer vision applications for an image analysis. Total Variation based image restoration was explicitly used to restore the denoised sharp and plane edge discontinuous in an image while preserving edge details from the noise.

The paper in [1] gives the min-max problem as:

$\left\{\underset{\mathrm{m}}{\min } \int_{n}^{\infty}|\nabla b| x\|b-i\|_{2}^{2} \leq w^{2}\right.$

in eq (1), $\Omega$ gives the bounded image values of the denoised region, $\mathrm{i}$ represent the input image, $\mathrm{v}$ is the noise variance in the image. The objective function in the eq. (1) is the Euclidean L2 semi-norm function of b. Eq. (1) fails at unconstrained minimization problems, to formulate the subsequent of (1), the authors [2] formulates the TV model as:

$\left\{\int_{\mathrm{L}}^{m i n} P(b) x=\int_{n}^{30}|\nabla b| d x+\frac{a b}{n}\|b-i\|_{2}^{2}\right.$

by choosing proper $\lambda$ value, the unconstrained minimization problem can be eliminated. The $\lambda$ value can be maximized using a second order dual $\lambda$ formulation [8], [4] and [5] as:

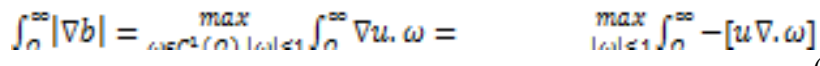

in eq (3) the $\omega$ give the range value extents as $\Omega \rightarrow \breve{I} 2$. The $\omega$ value define TV regularization requires the value of $b$ only, to have dual $\lambda$ formulation. The dual $\lambda$ formulation needs a smooth edge denoising using bounded unit plane edge variations. In fact, this is the definition of proposed unit plane edge method for the space of bounded unit plane edge functions.

To derive the proposed unit plane edge method, the dual $\lambda$ formulation with TV model for edge denoising is associated with the bounded value and minimum-and-maximum edge values using integrate and unit process, and becomes the following equation in the form:

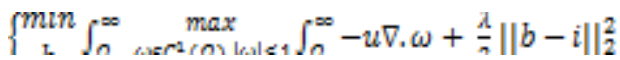

where $(\mathrm{u}, \mathrm{w})$ are $\mathrm{p}-\mathrm{d}$ variables. The integration and unit process allows extracting the edge values to associate a minimization process, which reduces the blur intensity across them and allows the unit value representation to associate a maximization process, which increases the dual $\lambda$ formulation effectively. The minimumand-maximum edge values allows to interchange the edge unit values, to result

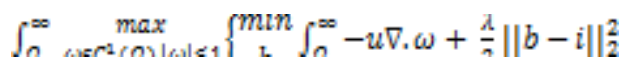

The unit value representation is as follows:

$b=i+\frac{1}{a} \nabla-\omega$

resulting to following unit plane edge formulation:

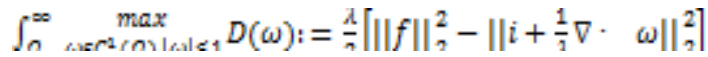

the unit plane edge formulation method equivalent is:

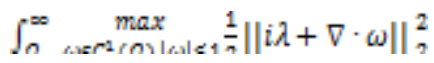

For a p-d unit plane edge variable $(\mathrm{u}, \mathrm{w})$, ON-OFF Slope algorithm is defined through two steps, the Slope variation between $\mathrm{ON}$ and $\mathrm{ON}-\mathrm{OFF}$ edge denoised image values and ON-OFF Slope representation. The first step can solve the dual $\lambda$ formulation for differences in the primal and dual edge method, using the

$R\left(u_{s} w\right)=\operatorname{Pr}(u)_{O N}^{O S F}-D u(w)_{O N}^{O S F}$

The objectives of first step are:

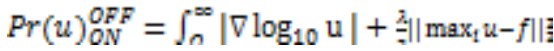

And

$D u(W)=\frac{\pi}{\pi}\left[\|f\|_{2}^{2}-\left\|\frac{1}{\pi} \nabla^{*} \omega+f\right\|_{2}^{2}\right]$

The Slope variation bounds the ON and ON-OFF edges to optionally of the p-d objectives, is given as:

$R\left(u_{w} w\right)=\int_{\Lambda}^{\mathrm{s}}\left(\left|\nabla \log _{10} \mathrm{u}\right|-\nabla \log \mathrm{u} w\right)+\frac{d}{2} \| \frac{1}{2} \nabla \cdot \omega+f-$ $u \|_{2}^{2}$

In Slope variation, if $\mathrm{u}$ and $\mathrm{w}$ are denoised specifics, the $\mathrm{ON}$ and $\mathrm{ON}-\mathrm{OFF}$ edges are related to primal and dual problem, respectively results

$0 \leq \operatorname{Pr}(u)-0^{*} \leq R\left(u_{w} w\right)$

$0 \leq 0^{*}-D u(w) \leq R(u, w)$

where $O^{*}$ is $\mathrm{p}-\mathrm{d} \lambda$ value. The primal $\lambda$ variable $\mathrm{u}$ is calculated using,

$\lambda_{T V}(u)=\sum_{1 \text { si.isn } f} f+\frac{1}{\mathfrak{a}} \nabla \cdot\left\|R\left(u_{w} W\right)_{\text {i. i }}\right\|$

$\&$ the dual $\lambda$ variable $w$ is calculated using,

$\lambda_{T V}(w)=\left(\nabla^{-}-\omega\right)_{i, j}=\omega_{(i, i) n+w^{i}} 1 \leq i_{i j} \leq n$

The second step ON-OFF Slope is called in the following way:

Table 1: ON-OFF Slope approach

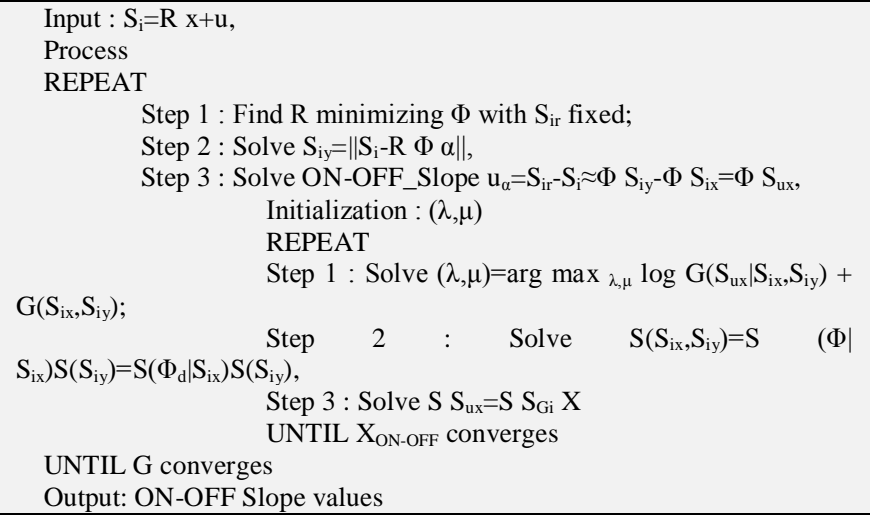

where $\mathrm{x}$ is the original image, $\mathrm{i}$ is the observed image for observation of ON-OFF and ON pixels, $\mathrm{S}_{\mathrm{G}}$ is the pixel interspacing factor, $\Phi$ is the edge artifact, $S_{\text {ir }}$ is the reconstructed image, $S_{\text {iy }}$ is the predicted image, $S_{i x}$ is the inter space image $\alpha$ is the slope variation factor, $\mu$ is image pixel state and $\mathrm{S}$ denotes the image $\mathrm{ON}$ OFF pixels slope values near to the primal and dual $\lambda$ estimations. The proposed ON-OFF Slope method provides the equality values of $\mathrm{ON}$ and $\mathrm{ON}-\mathrm{OFF}$ estimation values, which are global variables 
for dual $\lambda$ formulation, thus the proposed image restoration analysis in providing a solution to $\mathrm{R}(\mathrm{u}, \mathrm{w})$ with fulfilling the ON-OFF and $\mathrm{ON}$ image object pixels using inter spacing providing proper interdependency in unit plane edges.

Proposed algorithms, in Table 1, would lead to a good edge representation solution to image reconstruction analysis in general. Proposed algorithm is very efficient in reliability and effective for image analysis.

Eq. 12 refers to the denoised ON-OFF pixel image model by approximating the second term under the assumption that interspacing, proposed method can retrieve $\mathrm{Su}_{\mathrm{x}}$ in to the product of the $\mathrm{ON}$ OFF pixel estimators, such equation is,

$$
\begin{aligned}
& \text { Slope }(\varphi)=S(\text { Six, Siy })=S_{\text {ON }}(\varphi \mid \text { Six }) S(\text { Siy })= \\
& S_{\text {OFF }}(\varphi d \mid \text { Six }) S(\text { Siy })
\end{aligned}
$$

where $\Phi_{\mathrm{d}}=\Phi \mid \mathrm{S}_{\mathrm{ix}}-\Phi$, defines the deviation between unit edge ON and ON-OFF image pixels. Such restoration can be viewed as a level of proposed algorithm strategy, so $\Phi_{\mathrm{d}}$ is approximately independent from $\Phi \mid \mathrm{S}_{\mathrm{ix}}$

\section{Results and Discussions}

Proposed algorithm is tested with Barbara, Building, Lighthouse, Text, Airplane, Girl, Lenna, Women and Boat gary scale test images $(256 \times 256)$. All images are tested with Gaussian white noise $=$ 50, 100 and 200. Proposed work is compared with LBW DWF [1], FD WF [2], EWF [3], ATV [4] and NLM [5].

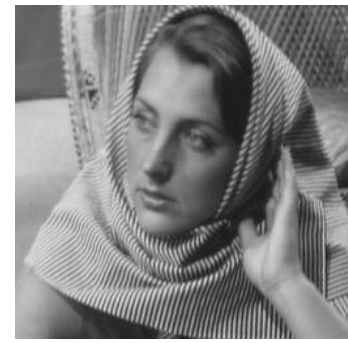

(a) Barbara

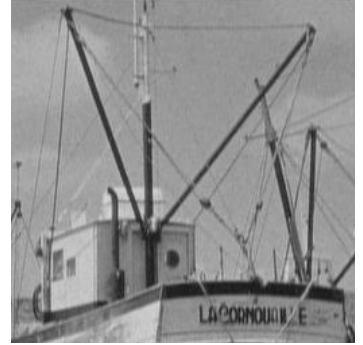

(b) Boat

Fig 1: 2D images considered for proposed work analysis.

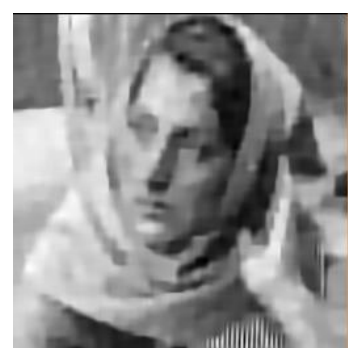

LBW DWF [1]

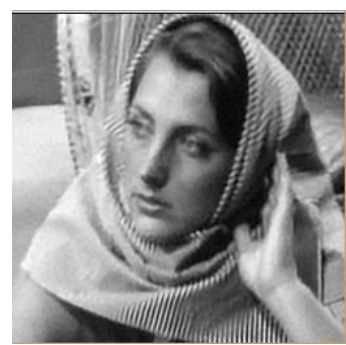

EWF [3]

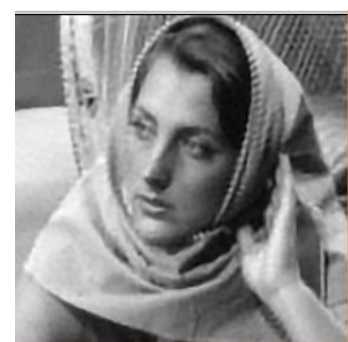

FD WF [2]

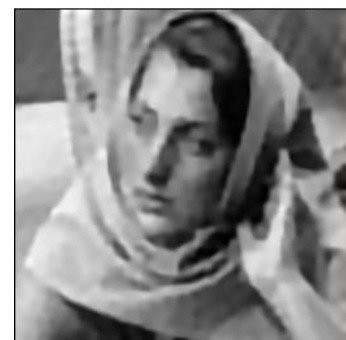

ATV [4]

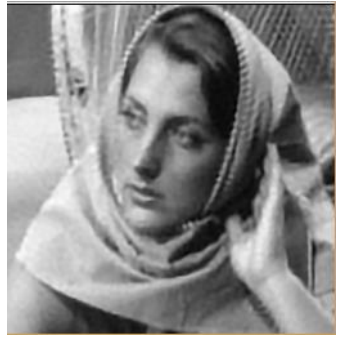

NLM [5]

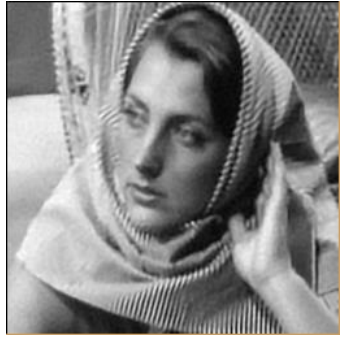

Proposed
Fig. 2 : Restoration comparison of Barbara with low noise level ( =50)

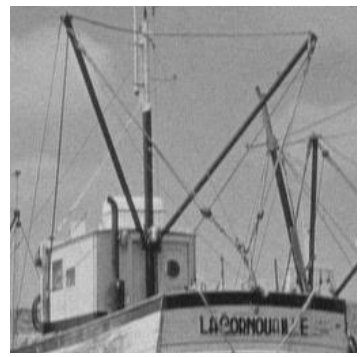

LBW DWF [1]

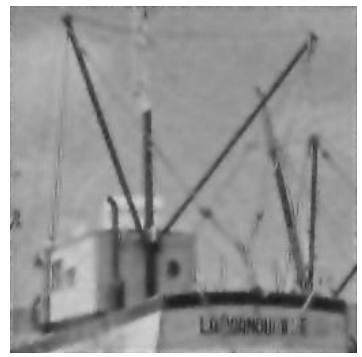

EWF [3]

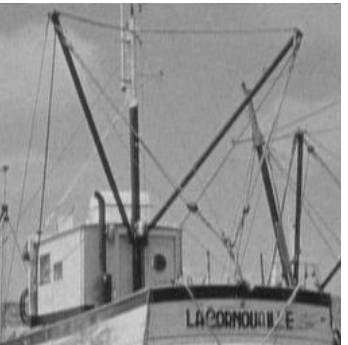

NLM [5]

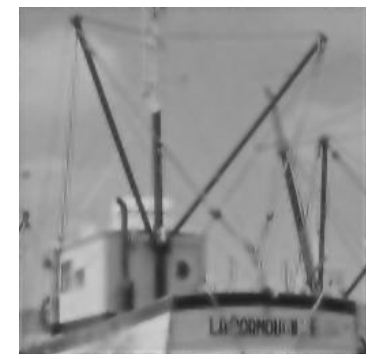

FD WF [2]

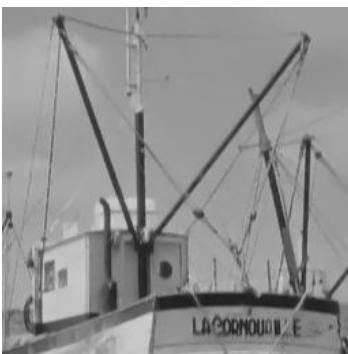

ATV [4]

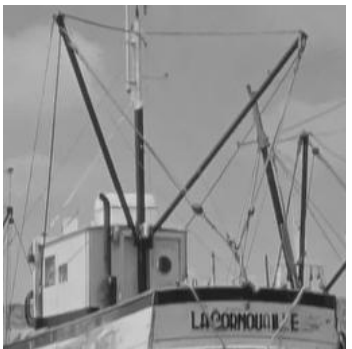

Proposed
Fig. 3: Restoration comparison of Boat with low noise level ( $=50$ )

\subsection{Comparative Image Reconstruction Results:}

Figure 1 shows the input images considered for visual image reconstruction comparison. Figure 2 shows the image restoration comparison of input image Barbara with low noise level $(=50)$ between comparative work and proposed work. Figure 3 shows the image restoration comparison of input image Boat with low noise level $(=50)$ between comparative work and proposed work. 


\subsection{Comparative Image Reconstruction Quality Results}

Table 2 and 3 give the performance evaluation in PSNR and MSSIM for Gaussian white noise $=50$, between compared and proposed method. Table 4 give the average time execution comparison for Gaussian white noise $=50,100$ and 200 between compared and proposed method.

Table 2: Performance comparison in PSNR ( =50)

\begin{tabular}{|c|c|c|c|c|c|c|c|c|c|}
\hline $\begin{array}{l}\text { Test } \\
\text { Im- } \\
\text { ages }\end{array}$ & $\begin{array}{l}\text { प्र } \\
\stackrel{\circ}{=}\end{array}$ & 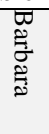 & 芯. & 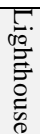 & $\overrightarrow{0}$ & 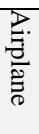 & $\stackrel{?}{0}$ & () & 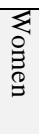 \\
\hline $\begin{array}{l}\text { PSN } \\
\mathrm{R} \\
(\mathrm{dB})\end{array}$ & 60 & 65 & 64 & 62 & 64 & 62 & 65 & 64 & 65 \\
\hline
\end{tabular}

Table 3: Performance comparison in $\operatorname{MSSIM}(100 \%)(=50)$

\begin{tabular}{|c|c|c|c|c|c|c|}
\hline 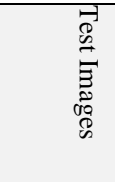 & 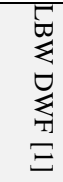 & $\begin{array}{l}\text { Ty } \\
\sum \\
\text { I } \\
N\end{array}$ & $\begin{array}{l}\text { 留 } \\
\text { T] } \\
\text { w }\end{array}$ & $\begin{array}{l}\stackrel{D}{3} \\
\frac{D}{ \pm}\end{array}$ & $\begin{array}{l}Z \\
3 \\
\frac{\pi}{\pi}\end{array}$ & 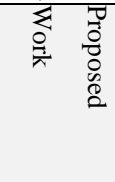 \\
\hline Barbara & 67 & 95 & 96 & 94 & 97 & 98 \\
\hline Building & 62 & 96 & 96 & 96 & 96 & 97 \\
\hline $\begin{array}{l}\text { Light- } \\
\text { house }\end{array}$ & 68 & 91 & 94 & 90 & 93 & 96 \\
\hline Text & 68 & 95 & 96 & 95 & 93 & 97 \\
\hline Airplane & 81 & 89 & 92 & 95 & 94 & 96 \\
\hline Girl & 82 & 91 & 92 & 91 & 93 & 94 \\
\hline Lenna & 83 & 91 & 94 & 96 & 96 & 97 \\
\hline Women & 81 & 91 & 94 & 95 & 95 & 96 \\
\hline Boat & 79 & 93 & 94 & 93 & 96 & 97 \\
\hline
\end{tabular}

\begin{tabular}{|c|c|c|c|c|c|c|c|}
\hline 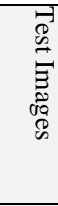 & 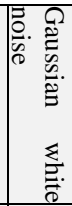 & $\begin{array}{l}\text { E } \\
\sum \\
\Xi \\
\Xi \\
\Xi \\
\Xi\end{array}$ & $\begin{array}{l}\text { T] } \\
\sum \\
\text { I } \\
\underline{N}\end{array}$ & 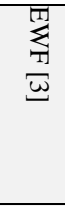 & \begin{tabular}{l}
$\geq$ \\
\multirow{2}{*}{} \\
\pm
\end{tabular} & $\begin{array}{l}z \\
z \\
z \\
\pi\end{array}$ & 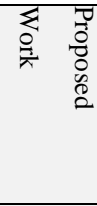 \\
\hline \multirow{3}{*}{$\begin{array}{l}\text { Bar- } \\
\text { bara }\end{array}$} & 50 & 0.60 & 0.31 & 0.93 & 23.33 & 67.31 & 0.28 \\
\hline & 100 & 0.56 & 0.31 & 0.92 & 66.17 & 68.60 & 0.21 \\
\hline & 225 & 0.57 & 0.30 & 0.93 & $\begin{array}{l}751.4 \\
9\end{array}$ & 67.82 & 0.20 \\
\hline \multirow[t]{3}{*}{ Boat } & 50 & 0.58 & 0.30 & 0.89 & 21.01 & 65.22 & 0.26 \\
\hline & 100 & 0.54 & 0.30 & 0.80 & 64.52 & 66.89 & 0.19 \\
\hline & 225 & 0.55 & 0.28 & 0.91 & $\begin{array}{l}721.5 \\
5\end{array}$ & 68.23 & 0.18 \\
\hline
\end{tabular}

\section{Conclusions}

Proposed an image restoration analysis in unit plane edge that is effective for denoising the image fine edges by reducing the noise in the reconstructed images. From the survey, it is clear that proposed method is fast for restoring images that are efficient in edge details.

\section{References}

[1] E. Ercelebi and S. Koc, "Lifting-based wavelet domain adaptive Wiener filter for image enhancement" IEE Proc. Vis. Image and Signal Processing, vol.153, no.1, pp. 31-36, Feb. 2006.

[2] S. Suhaila and T. Shimamura "Power spectrum estimation method for image denoising by frequency domain Wiener filter " in Proc. IEEE Int. Conf. Computer and Automation Engineering, Singapore, 2010, pp. 608-612.

[3] S. Suhaila, and T. Shimamura, "Image Restoration Based on Edgemap and Wiener Filter for Preserving Fine Details and Edges", International Journal Of Circuits, Systems And Signal Processing, Issue 6, Volume 5,pp-618-626, 2011.

[4] G. Gilboa, N. Sochen, and Y. Zeevi "Texture preserving variational denoising using an adaptive fidelity term " in Proc. IEEE Workshop Variational and Level Set Methods in Computer Vision, Nice, 2003 pp.137-144.

[5] Buades, B. Coll and J M Morel "A non-local algorithm for image denoising " in Proc. IEEE Int. Conf. Computer Vision and Pattern Recognition, San Diego, 2005, vol.2, pp. 60-65.

[6] W. Dong, G. Shi, Y. Ma, and X. Li, "Image Restoration via Simultaneous Sparse Coding: Where Structured Sparsity Meets Gaussian Scale Mixture," International Journal of Computer Vision (IJCV), vol. 114 , no. 2 , pp. 217-232, Sep. 2015.

[7] Jian Zhang, Debin Zhao, Wen Gao, "Group-based Sparse Representation for Image Restoration," IEEE Transactions on Image Processing, 15-July-2015.

[8] Vardan Papyan and Michael Elad, Multi-Scale Patch-Based Image Restoration, IEEE Transactions on Image Processing, vol. 25, no. 1, pages 249-261, January 2016.

[9] Uwe Schmidt and Stefan Roth, "Shrinkage Fields for Effective Image Restoration," IEEE Conference on Computer Vision and Pattern Recognition (CVPR), Columbus, Ohio, June 2014.

[10] Kheradmand and P. Milanfar, "A general framework for regularized, similarity-based image restoration," IEEE Transactions on Image Processing, vol. 23, no. 12, pp. 5136-5151, Dec 2014.

[11] C. H. Hsieh, P. C. Huang and S. Y. Hung, "Noisy image restoration based on boundary resetting BDND and median filtering with smallest window" WSEAS Trans. Signal Processing, vol.5, no.5, pp. 178-187, May 2009.

[12] V. Gui and C Caleanu "On the effectiveness of multiscale mode filters in edge preserving " in Proc. WSEAS Int. Conf. Systems, Rodos Island, 2009, pp. 190-195.

[13] S. Suhaila and T. Shimamura "Power spectrum estimation method for image denoising by frequency domain wiener filter " in Proc. IEEE Int. Conf. Computer and Automation Engineering, Singapore, 2010, pp. 608-612.

[14] Zhang Y, Pu Y-F, Hu J-R and Zhou J-L 2012 A class of fractional order variational image inpainting models. Appl. Math. Inf. Sc. Int. J. 2: 299-306

[15] M. V. Afonso, J. M. Bioucas-Dias, and M. A. T. Figueiredo, "An augmented lagrangian approach to the constrained optimization formulation of imaging inverse problems," Image Processing, IEEE Transactions on, vol. 20, no. 3, pp. 681 - 695, Mar. 2011

[16] Beck and M. Teboulle, "A fast iterative shrinkage-thresholding algorithm for linear inverse problems," SIAM Journal of Imaging Science, vol. 2, no. 1, pp. 183-202, Mar 2009.

[17] Y. Huang, M. Ng, and T. Zeng, "The convex relaxation method on deconvolution model with multiplicative noise," Commun. Comput. Phys., vol. 13, no. 4, pp. 1066-1092, 2013. 